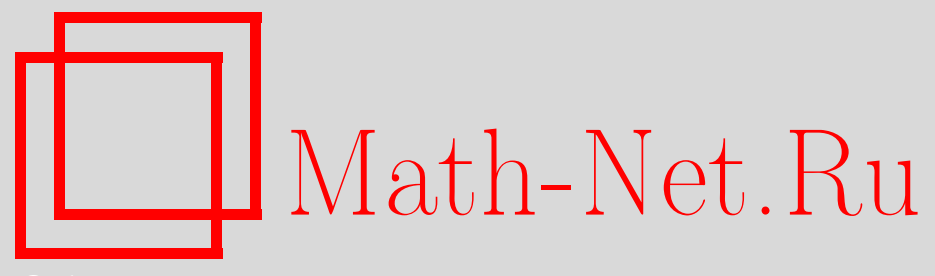

М. Дж. Марданов, К. Б. Мансимов, Н. Г. Абдуллаева, Необходимое условие оптимальности второго порядка в задачах управления, описываемых системой интегро-дифференциальных уравнений с запаздыванием, Вестн. Сам. гос. техн. ун-та. Сер. Физ.-мат. науки, 2018, номер 2, 254-268

DOI: https://doi.org/10.14498/vsgtu1597

Использование Общероссийского математического портала MathNet.Ru подразумевает, что вы прочитали и согласны с пользовательским соглашением

http: //www.mathnet.ru/rus/agreement

Параметры загрузки:

IP: 3.80 .181 .102

26 апреля 2023 г., 14:04:47

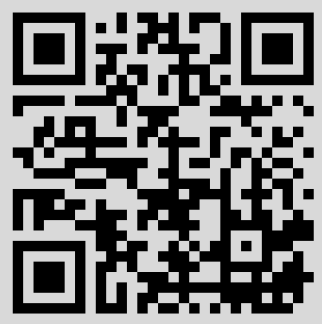


Вестн. Сам. гос. техн. ун-та. Сер. Физ.-мат. науки. 2018. Т. 22, № 2 . С. $254-268$

ISSN: 2310-7081 (online), 1991-8615 (print)

doi) http://doi.org/10.14498/vsgtu1597

УДК 517.977.56

\title{
Необходимое условие оптимальности второго порядка в задачах управления, описываемых системой интегро-дифференциальных уравнений с запаздыванием
}

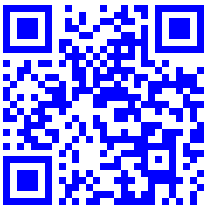

\author{
М. Дән. Марданов ${ }^{1}$, К. Б. Мансимов ${ }^{2,3}$, Н. Г. Абдуллаева \\ 1 Институт математики и механики НАН Азербайджана, \\ Азербайджан, AZ1141, Баку, ул. Б. Вахабзаде, 9. \\ 2 Бакинский государственный университет, \\ Азербайджан, AZ1148, Баку, ул. ак. 3 Халилова, 23. \\ 3 Институт систем управления НАН Азербайджана, \\ Азербайджан, AZ1141, Баку, ул. Б. Вахабзаде 9.
}

Аннотация

Рассматривается задача оптимального управления, описываемая системой интегро-дифференциальных уравнений типа Вольтерра с запаздывающим аргументом и многоточечным критерием качества. При предположении открытости области управления вычислены первая и вторая вариации критерия качества. Из равенства нулю первой вариации функционала качества вдоль оптимального процесса выведено необходимое условие оптимальности первого порядка в форме аналога уравнения Эйлера. Далее получено неявное необходимое условие оптимальности второго порядка, с помощью которого установлено довольно общее, но конструктивно проверяемое необходимое условие оптимальности второго порядка. Полученные результаты могут быть использованы для построения легко проверяемых необходимых условий оптимальности особых в классическом смысле управлений.

Ключевые слова: интегро-дифференциальное уравнение типа Вольтерра, оптимальное уравнение, необходимое условие оптимальности в интегральной форме, аналог уравнения Эйлера, классическая экстремаль, необходимое условие оптимальности второго порядка.

\section{Научная статья}

(2) (7) Контент публикуется на условиях лицензии Creative Commons Attribution 4.0 International (https://creativecommons.org/licenses/by/4.0/deed.ru)

\section{Образец для цитирования}

Марданов М. Дж., М ан с и мов К. Б., Абдулл а ев а Н. Г. Необходимое условие оптимальности второго порядка в задачах управления, описываемых системой интегро-дифференциальных уравнений с запаздыванием // Вестн. Сам. гос. техн. ун-та. Сер. Физ.-мат. науки, 2018. Т. 22, № 2. C. 254-268. doi: 10.14498/vsgtu1597.

\section{Сведения об авторах}

Мисир Джумаил огль Марданов (D) http://orcid.org/0000-0003-3901-0719

доктор физико-математических наук, профессор, член-корреспондент НАНА; директор института ${ }^{1}$; e-mail: misir .mardanov@imm.az

Камиль Байрамали огль Мансимов (1) http://orcid.org/0000-0002-1518-2279

доктор физико-математических наук, профессор; заведующий кафедрой; каф. математической кибернетики ${ }^{2}$; руководитель лаборатории; лаб. управления в сложных динамических системах ${ }^{3}$; e-mail: kamilbmansimov@gmail.com

Ниса Гасан къзы Абдуллаева (1) http://orcid.org/0000-0002-8834-5278

аспирант; лаб. управления в сложных динамических системах ${ }^{3}$; e-mail: kmansimov@mail.ru 
Получение: 27 декабря 2017 г. / Исправление: 14 апреля 2018 г. Принятие: 11 июня 2018 г. / Публикация онлайн: 1 июля 2018 г.

Введение. Многие процессы из механики, биофизики и других областей описываются интегро-дифференциальными уравнениями типа Вольтерра (см., например, работы [1-10]).

В работах [5-8, 10-16 и др.] исследованы различные задачи оптимального управления, описываемые интегро-дифференциальными уравнениями типа Вольтерра. Найдены необходимые условия оптимальности первого порядка, доказаны теоремы существования оптимальных управлений.

В предлагаемой работе рассматривается одна задача оптимального управления, описываемая системой интегро-дифференциальных уравнений типа Вольтерра с запаздыванием. При предположении открытости области управления получены необходимые условия оптимальности первого и второго порядков.

1. Постановка задачи. Рассмотрим управляемый процесс, описываемый системой интегро-дифференциальных уравнений типа Вольтерра с запаздыванием

$$
\begin{aligned}
\dot{x}=f(t, x(t), x(h(t)) & , u(t))+ \\
& +\int_{t_{0}}^{t} K(t, \tau, x(\tau), x(h(\tau)), u(\tau)) d \tau, \quad t \in T=\left[t_{0}, t_{1}\right],
\end{aligned}
$$

с начальным условием

$$
x(t)=a(t), \quad t \in E_{t_{0}}=\left[h\left(t_{0}\right), t_{0}\right] .
$$

Здесь $f(t, x, y, u)(K(t, \tau, x, y, u))$ - заданная $n$-мерная вектор-функция, непрерывная в $T \times \mathbb{R}^{n} \times \mathbb{R}^{n} \times \mathbb{R}^{r}\left(T \times T \times \mathbb{R}^{n} \times \mathbb{R}^{n} \times \mathbb{R}^{r}\right)$ вместе с частными производными по переменным $x, y, u$ до второго порядка включительно; $h(t)(h(t)<t)$ - заданная непрерывно дифференцируемая скалярная функция, причем $\dot{h}(t)>0 ; a(t), t \in E_{t_{0}}$ - заданная непрерывная начальная вектор-функция; $u(t)-r$-мерная кусочно-непрерывная (с конечным числом точек разрыва первого рода) вектор-функция управляющих воздействий со значениями из заданного непустого, ограниченного и открытого множества $U \subset \mathbb{R}^{r}:$

$$
u(t) \in U \subset \mathbb{R}^{r}, \quad t \in T .
$$

Такие управляющие функции назовем допустимыми.

Предполагается, что каждому допустимому управлению $u(t)$ соответствует единственное решение $x(t)$ (в смысле, например, $[6,8,10]$ ) начальной задачи (1), (2).

На решениях системы (1), (2), порожденных всевозможными допустимыми управлениями, определим многоточечный функционал

$$
S(u)=\varphi\left(x\left(T_{1}\right), x\left(T_{2}\right), \ldots, x\left(T_{k}\right)\right) .
$$


Здесь $T_{i} \in\left(t_{0}, t_{1}\right]$ - заданные точки, причем $t_{0}<T_{1}<T_{2}<\ldots,<T_{k} \leqslant t_{1}$, а $\varphi\left(a_{1}, a_{2}, \ldots, a_{k}\right)$ - заданная дважды непрерывно дифференцируемая в $\mathbb{R}^{n k}$ скалярная функция.

Рассмотрим задачу о минимуме функционала (4) при ограничениях (1)-(3).

Допустимое управление $u(t)$, являющееся решением поставленной задачи, назовем оптимальным управлением, а соответствующий процесс $(u(t), x(t))$ оптимальным процессом.

Как видно, многоточечный функционал (4) является обобщением терминального функционала.

Перейдем к выводу необходимых условий оптимальности.

2. Вариации функционалов и необходимые условия оптимальности. Пусть $u(t)$ и $\bar{u}(t)=u(t)+\Delta u(t)$ - два допустимых управления. Через $x(t)$ и $\bar{x}(t)=x(t)+\Delta x(t)$ обозначим соответствующие им решения задачи (1), (2) и вычислим приращение функционала качества:

$$
\begin{aligned}
\Delta S(u)=S(\bar{u}) & -S(u)= \\
& =\varphi\left(\bar{x}\left(T_{1}\right), \bar{x}\left(T_{2}\right), \ldots, \bar{x}\left(T_{k}\right)\right)-\varphi\left(x\left(T_{1}\right), x\left(T_{2}\right), \ldots, x\left(T_{k}\right)\right) .
\end{aligned}
$$

Введем функцию Гамильтона-Понтрягина

$$
H(t, x, y, \psi(t))=\psi^{\prime}(t) f(t, x, y, u)+\int_{t}^{t_{1}} \psi^{\prime}(\tau) K(\tau, t, x, y, u) d \tau,
$$

где предполагается $y(t) \equiv x(h(t))$. Здесь $\psi=\psi(t)-n$-мерная вектор-функция сопряженных переменных, являющаяся решением задачи (сопряженная система)

$$
\begin{aligned}
\psi(t)=\int_{t}^{t_{1}} H_{x}(\tau, & x(\tau), y(\tau), u(\tau), \psi(\tau)) d \tau- \\
& \quad-\sum_{i=1}^{k} \alpha_{i}(t) \frac{\partial}{\partial a_{i}} \varphi\left(x\left(T_{1}\right), x\left(T_{2}\right), \ldots, x\left(T_{k}\right)\right)+ \\
& +\int_{t}^{t_{1}} H_{y}(r(\tau), x(r(\tau)), x(\tau), u(r(\tau)), \psi(r(\tau))) \dot{r}(\tau) d \tau,
\end{aligned}
$$

где $\psi(t) \equiv 0$ при $t>t_{1}, \alpha(t)$ - характеристическая функция отрезка $\left[t_{0}, T_{i}\right]$, а $r(t)$ - функция обратная к $h(t)$.

При некоторых предположениях (см., например, $[8,10])$ можно доказать существование кусочно-непрерывного решения задачи (6).

Приращение (5) функционала качества при помощи формулы Тейлора представляется в виде

$$
\begin{aligned}
\Delta S(u) & =\sum_{i=1}^{k} \frac{\partial}{\partial a_{i}} \varphi^{\prime}\left(x\left(T_{1}\right), x\left(T_{2}\right), \ldots, x\left(T_{k}\right)\right) \Delta x\left(T_{i}\right)+ \\
& +\frac{1}{2} \sum_{i=1}^{k} \sum_{i=1}^{k} \Delta x^{\prime}\left(T_{i}\right) \frac{\partial^{2}}{\partial a_{i} \partial a_{j}} \varphi\left(x\left(T_{1}\right), x\left(T_{2}\right), \ldots, x\left(T_{k}\right)\right) \Delta x\left(T_{j}\right)+
\end{aligned}
$$




$$
\begin{gathered}
+o_{1}\left(\left(\sum_{i=1}^{k} \Delta\left\|x\left(T_{i}\right)\right\|\right)^{2}\right)+ \\
+\int_{t_{0}}^{t_{1}} \psi^{\prime}(t) \Delta \dot{x}(t) d t+\int_{t_{0}}^{t_{1}} H_{x}^{\prime}(t, x(t), y(t), u(t), \psi(t)) \Delta x(t) d t- \\
-\int_{t_{0}}^{t_{1}} H_{y}^{\prime}(t, x(t), y(t), u(t), \psi(t)) \Delta y(t) d t- \\
-\frac{1}{2} \int_{t_{0}}^{t_{1}}\left[\begin{array}{l}
t_{1} \\
\Delta x^{\prime}(t)
\end{array}\right. \\
+H_{x x}(t, x(t), y(t), u(t), \psi(t)) \Delta x(t)+ \\
+\Delta x^{\prime}(t) H_{x y}(t, x(t), y(t), u(t), \psi(t)) \Delta y(t)+ \\
+\Delta y^{\prime}(t) H_{y x}(t, x(t), y(t), u(t), \psi(t)) \Delta x(t)+ \\
+\Delta y^{\prime}(t) H_{y y}(t, x(t), y(t), u(t), \psi(t)) \Delta y(t)+ \\
+2 \Delta u^{\prime}(t) H_{u x}(t, x(t), y(t), u(t), \psi(t)) \Delta x(t)+ \\
+2 \Delta u^{\prime}(t) H_{u y}(t, x(t), y(t), u(t), \psi(t)) \Delta y(t)+ \\
\left.+\Delta u^{\prime}(t) H_{u u}(t, x(t), y(t), u(t), \psi(t)) \Delta u(t)\right] d t- \\
t_{t_{0}}^{t_{1}} o_{2}\left([\|\Delta x(t)\|+\|y(t)\|+\|\Delta(t)\|]^{2}\right) d t
\end{gathered}
$$

Выполним некоторые преобразования над слагаемыми формулы (7):

$$
\begin{gathered}
\int_{t_{0}}^{t_{1}} H_{y}^{\prime}(t, x(t), y(t), u(t), \psi(t)) \Delta x(h(t)) d t= \\
\quad=\int_{h\left(t_{0}\right)}^{h\left(t_{1}\right)} H_{y}^{\prime}(r(t), x(r(t)), x(t), u(r(t)), \psi(r(t))) \Delta x(t) \dot{r}(t) d t= \\
=\int_{t_{0}}^{h\left(t_{1}\right)} H_{y}^{\prime}(r(t), x(r(t)), x(t), u(r(t)), \psi(r(t))) \dot{r}(t) \Delta x(t) d t= \\
=\int_{t_{0}}^{t_{1}}\left[\int_{t}^{t_{1}} H_{y}^{\prime}(r(\tau), x(r(\tau)), x(\tau), u(r(\tau)), \psi(r(\tau))) \dot{r}(\tau) d \tau\right] \Delta \dot{x}(t) d t,
\end{gathered}
$$

$$
\begin{aligned}
& \int_{t_{0}}^{t_{1}} H_{x}^{\prime}(t, x(t), y(t), u(t), \psi(t)) \Delta x(t) d t= \\
& =\int_{t_{0}}^{t_{1}}\left[\int_{t}^{t_{1}} H_{x}^{\prime}(\tau, x(\tau), x(h(\tau)), u(\tau), \psi(\tau)) d \tau\right] \Delta \dot{x}(t) d t
\end{aligned}
$$

$$
\sum_{i=1}^{k} \frac{\partial}{\partial a_{i}} \varphi^{\prime}\left(x\left(T_{1}\right), x\left(T_{2}\right), \ldots, x\left(T_{k}\right)\right) \Delta x\left(T_{i}\right)=
$$




$$
=\int_{t_{0}}^{t_{1}} \sum_{i=1}^{k} \alpha_{i}(t) \frac{\partial}{\partial a_{i}} \varphi\left(x\left(T_{1}\right), x\left(T_{2}\right), \ldots, x\left(T_{k}\right)\right) \Delta \dot{x}(t) d t .
$$

С учетом тождеств (8)-(10) из (7) получаем, что если $\psi(t)$ является решением сопряженной системы (6), то приращение функционала качества может быть представлено в виде

$$
\begin{aligned}
& \Delta S(u)=-\int_{t_{0}}^{t_{1}} H_{u}^{\prime}(t, x(t), y(t), u(t), \psi(t)) \Delta u(t) d t+ \\
& +\frac{1}{2} \sum_{i, j=1}^{k} \Delta x^{\prime}\left(T_{i}\right) \frac{\partial^{2}}{\partial a_{i} \partial a_{j}} \varphi\left(x\left(T_{1}\right), x\left(T_{2}\right), \ldots, x\left(T_{k}\right)\right) \Delta x\left(T_{j}\right)- \\
& -\frac{1}{2} \int_{t_{0}}^{t_{1}}\left[\Delta x^{\prime}(t) H_{x x}(t, x(t), y(t), u(t), \psi(t)) \Delta x(t)+\right. \\
& +\Delta y^{\prime}(t) H_{y x}(t, x(t), y(t), u(t), \psi(t)) \Delta x(t)+ \\
& +\Delta x^{\prime}(t) H_{x y}(t, x(t), y(t), u(t), \psi(t)) \Delta y(t)+ \\
& \left.+\Delta y^{\prime}(t) H_{y y}(t, x(t), y(t), u(t), \psi(t)) \Delta y(t)\right] d t- \\
& -2 \int_{t_{0}}^{t_{1}}\left[\Delta u^{\prime}(t) H_{u x}(t, x(t), y(t), u(t), \psi(t)) \Delta x(t)+\right. \\
& \left.+\Delta u^{\prime}(t) H_{u y}(t, x(t), y(t), u(t), \psi(t)) \Delta y(t)\right] d t- \\
& -\frac{1}{2} \int_{t_{0}}^{t_{1}} \Delta u^{\prime}(t) H_{u u}(t, x(t), y(t), u(t), \psi(t)) \Delta u(t) d t+\eta_{1}(u ; \Delta u),
\end{aligned}
$$

где обозначено

$\eta_{1}(u ; \Delta u)=o_{1}\left(\left(\sum_{i=1}^{k} \Delta\left\|x\left(T_{i}\right)\right\|\right)^{2}\right)-\int_{t_{0}}^{t_{1}} o_{2}\left([\|\Delta x(t)\|+\|\Delta y(t)\|+\|\Delta u(t)\|]^{2}\right) d t$.

Пусть $\varepsilon-$ произвольное достаточно малое по абсолютной величине число, а $\delta u(t)$ - произвольная кусочно-непрерывная $r$-мерная вектор-функция со значениями из $R^{r}$ (допустимая вариация управления).

В силу открытости области управления специальное приращение допустимого управления $u(t)$ можно определить по формуле

$$
\Delta u_{\varepsilon}(t)=\varepsilon \delta u(t), \quad t \in T .
$$

Через $\Delta x_{\varepsilon}(t)$ обозначим специальное приращение траектории $x(t)$, отвечающее специальному приращению (12) управления $u(t)$.

Пусть по определению $u_{\varepsilon}(t)=u(t)+\Delta u_{\varepsilon}(t), x_{\varepsilon}(t)=x(t)+\Delta x_{\varepsilon}(t)$.

Из $(1),(2)$ следует, что $\Delta x_{\varepsilon}(t)$ является решением следующей линеаризованной задачи:

$$
\Delta \dot{x}_{\varepsilon}(t)=f_{x}(t, x(t), x(h(t)), u(t)) \Delta x_{\varepsilon}(t)+
$$




$$
\begin{gathered}
+f_{y}(t, x(t), x(h(t)), u(t)) \Delta y_{\varepsilon}(t)+ \\
+f_{u}(t, x(t), x(h(t)), u(t)) \Delta u_{\varepsilon}(t)+ \\
+\int_{t_{0}}^{t}\left[K_{x}(t, \tau, x(\tau), x(h(\tau)), u(\tau)) \Delta x_{\varepsilon}(\tau)+\right. \\
+K_{y}(t, \tau, x(\tau), x(h(\tau)), u(\tau)) \Delta y_{\varepsilon}(\tau)+ \\
\left.+K_{u}(t, \tau, x(\tau), x(h(\tau)), u(\tau)) \Delta u_{\varepsilon}(\tau)\right] d \tau+ \\
+o_{3}\left(\left\|\Delta x_{\varepsilon}(\tau)\right\|+\left\|\Delta y_{\varepsilon}(\tau)\right\|+\left\|\Delta u_{\varepsilon}(\tau)\right\|\right)+ \\
+\int_{t_{0}}^{t_{1}} o_{4}\left(\left\|\Delta x_{\varepsilon}(\tau)\right\|+\left\|\Delta y_{\varepsilon}(\tau)\right\|+\left\|\Delta u_{\varepsilon}(\tau)\right\|\right) d \tau,
\end{gathered}
$$

где

$$
\Delta x_{\varepsilon}(t) \equiv 0, \quad t \in E_{t_{0}} .
$$

С применением (13), (14) доказывается

Лемма. Для $\Delta x_{\varepsilon}(t)$ справедливо следующее разложение:

$$
\Delta x_{\varepsilon}(t)=\varepsilon \delta x(t)+o(\varepsilon ; t),
$$

где $\delta x(t)$ (вариачия траектории) является решением задачи

$$
\begin{aligned}
& \delta \dot{x}_{\varepsilon}(t)=f_{x}(t, x(t), x(h(t)), u(t)) \delta x(t)+f_{y}(t, x(t), x(h(t)), u(t)) \delta y(t)+ \\
+ & \int_{t_{0}}^{t}\left[K_{x}(t, \tau, x(\tau), x(h(\tau)), u(\tau)) \delta x(\tau)+K_{y}(t, \tau, x(\tau), x(h(\tau)), u(\tau)) \delta y(\tau)\right] d \tau+ \\
& +f_{u}(t, x(t), x(h(t)), u(t)) \delta u(t)+\int_{t_{0}}^{t} K_{u}(t, \tau, x(\tau), x(h(\tau)), u(\tau)) \delta u(\tau) d \tau, \quad \text { (16) }
\end{aligned}
$$

где

$$
\delta x_{\varepsilon}(t) \equiv 0, \quad t \in E_{t_{0}} .
$$

Уравнение (16) назовем уравнением в вариациях для рассматриваемой задачи.

Учитывая (12), (15), из формулы приращения (11) получаем справедливость разложения

$$
\begin{aligned}
\Delta S_{\varepsilon}(u)= & S(u+\varepsilon \delta u)-S(u)= \\
= & \varepsilon \int_{t_{0}}^{t_{1}} H_{u}^{\prime}(t, x(t), x(h(t)), u(t), \psi(t)) \delta u(t) d t+ \\
+ & \frac{\varepsilon^{2}}{2}\left\{\sum_{i, j=1}^{k} \delta x^{\prime}\left(T_{i}\right) \frac{\partial^{2}}{\partial a_{i} \partial a_{j}} \varphi\left(x\left(T_{1}\right), x\left(T_{2}\right), \ldots, x\left(T_{k}\right)\right) \delta x\left(T_{j}\right)-\right. \\
& \quad-\int_{t_{0}}^{t_{1}}\left[\delta x^{\prime}(t) H_{x x}(t, x(t), x(h(t)), u(t), \psi(t)) \delta x(t)+\right.
\end{aligned}
$$




$$
\begin{aligned}
& +\delta x^{\prime}(t) H_{x y}(t, x(t), x(h(t)), u(t), \psi(t)) \delta y(t)+ \\
& +\delta y^{\prime}(t) H_{y x}(t, x(t), x(h(t)), u(t), \psi(t)) \delta x(t)+ \\
& +\delta y^{\prime}(t) H_{y y}(t, x(t), x(h(t)), u(t), \psi(t)) \delta y(t)+ \\
& +2 \delta u^{\prime}(t) H_{u x}(t, x(t), x(h(t)), u(t), \psi(t)) \delta x(t)+ \\
& +2 \delta u^{\prime}(t) H_{u y}(t, x(t), x(h(t)), u(t), \psi(t)) \delta y(t)+ \\
& \left.\left.\quad+\delta u^{\prime}(t) H_{u u}(t, x(t), x(h(t)), u(t), \psi(t)) \delta u(t)\right] d t\right\} .
\end{aligned}
$$

Из разложения (18) в силу определения первой и второй вариаций производной (в классическом смысле) функционала (см., например, [7, 12, 13, 18, 19]) следует, что

$$
\delta^{1} S(u ; \delta u)=-\int_{t_{0}}^{t_{1}} H_{u}^{\prime}(t, x(t), x(h(t)), u(t), \psi(t)) \delta u(t) d t,
$$

$$
\begin{aligned}
\delta^{2} S(u ; \delta u)= & \sum_{i, j=1}^{k} \delta x^{\prime}\left(T_{i}\right) \frac{\partial^{2}}{\partial a_{i} \partial a_{j}} \varphi\left(x\left(T_{1}\right), x\left(T_{2}\right), \ldots, x\left(T_{k}\right)\right) \delta x\left(T_{j}\right)- \\
- & \int_{t_{0}}^{t_{1}}\left[\delta x^{\prime}(t) H_{x x}(t, x(t), x(h(t)), u(t), \psi(t)) \delta x(t)+\right. \\
& +\delta x^{\prime}(t) H_{x y}(t, x(t), x(h(t)), u(t), \psi(t)) \delta y(t)+ \\
& +\delta y^{\prime}(t) H_{y x}(t, x(t), x(h(t)), u(t), \psi(t)) \delta x(t)+ \\
& +\delta y^{\prime}(t) H_{y y}(t, x(t), x(h(t)), u(t), \psi(t)) \delta y(t)+ \\
& +2 \delta u^{\prime}(t) H_{u x}(t, x(t), x(h(t)), u(t), \psi(t)) \delta x(t)+ \\
& +2 \delta u^{\prime}(t) H_{u y}(t, x(t), x(h(t)), u(t), \psi(t)) \delta y(t)+ \\
& \left.\quad+\delta u^{\prime}(t) H_{u u}(t, x(t), x(h(t)), u(t), \psi(t)) \delta u(t)\right] d t .
\end{aligned}
$$

Известно (см., например, $[17,18])$, что в случае открытости области управления вдоль оптимального процесса для всех допустимых вариаций управления первая вариация функционала качества равна нулю, а вторая - неотрицательна:

$$
\delta^{1} S(u ; \delta u)=-\int_{t_{0}}^{t_{1}} H_{u}^{\prime}(t, x(t), x(h(t)), u(t), \psi(t)) \delta u(t) d t=0,
$$

$$
\begin{aligned}
\delta^{2} S(u ; \delta u)= & \sum_{i, j=1}^{k} \delta x^{\prime}\left(T_{i}\right) \frac{\partial^{2}}{\partial a_{i} \partial a_{j}} \varphi\left(x\left(T_{1}\right), x\left(T_{2}\right), \ldots, x\left(T_{k}\right)\right) \delta x\left(T_{j}\right)- \\
- & \int_{t_{0}}^{t_{1}}\left[\delta x^{\prime}(t) H_{x x}(t, x(t), x(h(t)), u(t), \psi(t)) \delta x(t)+\right. \\
& +\delta x^{\prime}(t) H_{x y}(t, x(t), x(h(t)), u(t), \psi(t)) \delta y(t)+
\end{aligned}
$$




$$
\begin{aligned}
& \quad+\delta y^{\prime}(t) H_{y x}(t, x(t), x(h(t)), u(t), \psi(t)) \delta x(t)+ \\
& +\delta y^{\prime}(t) H_{y y}(t, x(t), x(h(t)), u(t), \psi(t)) \delta y(t)+ \\
& +2 \delta u^{\prime}(t) H_{u x}(t, x(t), x(h(t)), u(t), \psi(t)) \delta x(t)+ \\
& +2 \delta u^{\prime}(t) H_{u y}(t, x(t), x(h(t)), u(t), \psi(t)) \delta y(t)+ \\
& \left.\quad+\delta u^{\prime}(t) H_{u u}(t, x(t), x(h(t)), u(t), \psi(t)) \delta u(t)\right] d t \geqslant 0
\end{aligned}
$$

для всех $\delta u(t) \in \mathbb{R}^{r}, t \in T$, где $\delta x(t)$ - решение уравнения в вариациях (16), (17). При помощи тождества (19) по схеме из [17] доказывается

ТЕОРема (АНАЛОГ УРАВНЕНИЯ ЭЙЛЕРА). ДЛя оптималъности допустимого управления $u(t)$ в задаче $(1)-(4)$ необходимо, чтобы соотношение

$$
H_{u}^{\prime}(\theta, x(\theta), x(h(\theta)), u(\theta), \psi(\theta))=0
$$

въполнялось для всех $\theta \in\left[t_{0}, t_{1}\right)$.

Здесь и далее $\theta \in\left[t_{0}, t_{1}\right), \theta \neq T_{i}$ - произвольная точка непрерывности управления $u(t)$.

Каждое допустимое управление, удовлетворяющее уравнению Эйлера (21), назовем классической экстремалью в задаче (1)-(4). Ясно, что оптимальное управление (если оно существует) находится среди классических экстремалей. Поэтому возникает необходимость в сужении множества классических экстремалей, а для этого надо иметь необходимые условия оптимальности второго порядка.

Неравенство (20) является неявным необходимым условием оптимальности второго порядка. Но, опираясь на него, можно получить необходимые условия оптимальности, выраженные непосредственно через параметры задачи (1)-(4).

В дальнейшем для простоты изложения будем использовать обозначения следующего типа:

$$
\begin{array}{ll}
H_{x}(t) \equiv H_{x}(t, x(t), y(t), u(t), \psi(t)), & H_{y}(t) \equiv H_{y}(t, x(t), y(t), u(t), \psi(t)), \\
H_{x y}(t) \equiv H_{x y}(t, x(t), y(t), u(t), \psi(t)), & H_{x x}(t) \equiv H_{x x}(t, x(t), y(t), u(t), \psi(t)), \\
H_{u}(t) \equiv H_{u}(t, x(t), y(t), u(t), \psi(t)), & H_{u u}(t) \equiv H_{u u}(t, x(t), y(t), u(t), \psi(t)), \\
f_{x}(t) \equiv f_{x}(t, x(t), x(h(t)), u(t)), & f_{y}(t) \equiv f_{y}(t, x(t), x(h(t)), u(t)), \\
f_{u}(t) \equiv f_{u}(t, x(t), x(h(t)), u(t)), & K_{x}(t, \tau) \equiv K_{x}(t, \tau, x(\tau), x(h(\tau)), u(\tau)), \\
K_{u}(t, \tau) \equiv K_{u}(t, \tau, x(\tau), x(h(\tau)), u(\tau)), & K_{y}(t, \tau) \equiv K_{y}(t, \tau, x(\tau), x(h(\tau)), u(\tau)) .
\end{array}
$$

Уравнение (16) является линейным неоднородным интегро-дифференциальным уравнением типа Вольтерра. На основе формулы об интегральном представлении решений линейных неоднородных интегро-дифференциальных уравнений типа Вольтерра (см., например, [9]) имеем

$$
\begin{aligned}
\delta x(t) & =\int_{t_{0}}^{t} F(t, s)\left[f_{u}(s) \delta u(s)+\int_{t_{0}}^{s} K_{u}(s, \tau) \delta u(\tau) d \tau\right] d s= \\
& =\int_{t_{0}}^{t} F(t, s) f_{u}(s) \delta u(s) d s+\int_{t_{0}}^{t}\left[\int_{t_{0}}^{s} F(t, s) K_{u}(s, \tau) \delta u(\tau) d \tau\right] d s=
\end{aligned}
$$




$$
=\int_{t_{0}}^{t}\left[F(t, s) f_{u}(s)+\int_{s}^{t} F(t, \tau) K_{u}(\tau, s) d \tau\right] \delta u(s) d s .
$$

Здесь $F(t, \tau)-(n \times n)$-матричная функция, являющаяся решением следующего интегро-дифференциального уравнения типа Вольтерра:

$$
\begin{aligned}
F_{t}(t, \tau)=f_{x}(t, x(t) & , x(h(t)), u(t)) F(t, \tau)+ \\
& \left.+f_{y}(t, x(t), x(h(t)), u(t)) F(h(t)), \tau\right)+ \\
+ & \int_{t_{0}}^{t} K_{x}(t, \tau, x(\tau), x(h(\tau)), u(\tau)) F(t, \tau) d \tau+ \\
& +\int_{t_{0}}^{t} K_{y}(t, \tau, x(\tau), x(h(\tau)), u(\tau)) F(h(t), \tau) d \tau
\end{aligned}
$$

где

$$
F(\tau, \tau)=E, \quad F(t, \tau)=0, \quad \tau>t
$$

В предположении

$$
Q(t, s)=F(t, s) f_{u}(s)+\int_{s}^{t} F(t, \tau) K_{u}(\tau, s) d \tau
$$

представление (22) записывается в виде

$$
\delta x(t)=\int_{t_{0}}^{t} Q(t, s) \delta u(s) d s .
$$

Отсюда в силу (23)

$$
\begin{aligned}
& \delta x(h(t))=\int_{t_{0}}^{t} Q(h(t), s) \delta u(s) d s \\
& \delta x\left(T_{i}\right)=\int_{t_{0}}^{t} \alpha_{i}(t) Q\left(T_{i}, s\right) \delta u(s) d s
\end{aligned}
$$

где, как и выше, $\alpha_{i}(t)$ - характеристическая функция отрезка $\left[t_{0}, T_{1}\right]$.

Используя формулы (24)-(26), преобразуем отдельные слагаемые в неравенстве (20):

$$
\begin{aligned}
& \int_{t_{0}}^{t_{1}} \delta u^{\prime}(t) H_{u x}(t) \delta x(t) d t=\int_{t_{0}}^{t_{1}}\left[\int_{t_{0}}^{t} \delta u^{\prime}(t) H_{u x}(t) Q(t, s) \delta u(s) d s\right] d t \\
& \int_{t_{0}}^{t_{1}} \delta u^{\prime}(t) H_{u y}(t) \delta x(h(t)) d t=\int_{t_{0}}^{t_{1}}\left[\int_{t_{0}}^{t} \delta u^{\prime}(t) H_{u y}(t) Q(h(t), s) \delta u(s) d s\right] d t,(28) \\
& \sum_{i, j=1}^{k} \delta x^{\prime}\left(T_{i}\right) \frac{\partial^{2}}{\partial a_{i} \partial a_{j}} \varphi\left(x\left(T_{1}\right), x\left(T_{2}\right), \ldots, x\left(T_{k}\right)\right) \delta x\left(T_{j}\right)=
\end{aligned}
$$




$$
\begin{aligned}
& =\int_{t_{0}}^{t_{1}} \int_{t_{0}}^{t_{1}} \sum_{i, j=1}^{k} \alpha_{i}(\tau) \alpha_{j}(s) \delta u^{\prime}(\tau) Q^{\prime}\left(T_{i}, \tau\right) \times \\
& \times \frac{\partial^{2}}{\partial a_{i} \partial a_{j}} Q\left(T_{j}, s\right) \varphi\left(x\left(T_{1}\right), x\left(T_{2}\right), \ldots, x\left(T_{k}\right)\right) \delta u(s) d s d \tau .
\end{aligned}
$$

Далее по схеме из [19-21] имеем

$$
\begin{aligned}
& \int_{t_{0}}^{t_{1}}\left[\delta x^{\prime}(t) H_{x x}(t) \delta x(t)+\delta x^{\prime}(t) H_{x y}(t) \delta y(t)+\delta y^{\prime}(t) H_{y y}(t) \delta y(t)\right] d t= \\
& =\int_{t_{0}}^{t_{1}} \int_{t_{0}}^{t_{1}} \delta u^{\prime}(\tau)\left\{\int _ { \operatorname { m a x } ( \tau , s ) } ^ { t _ { 1 } } \left[Q^{\prime}(t, \tau) H_{x x}(t) Q(t, s)+Q^{\prime}(h(t), \tau) H_{y x}(t) Q(t, s)+\right.\right. \\
& \left.\left.+Q^{\prime}(t, s) H_{x y}(t) Q(h(t), s)+Q^{\prime}(h(t), \tau) H_{y y}(t) Q(h(t), s)\right] d t\right\} \delta u(s) d s d \tau .
\end{aligned}
$$

Положим

$$
\begin{aligned}
& M(\tau, s)= \\
& =-\sum_{i, j=1}^{k} \alpha_{i}(\tau) \alpha_{j}(s) Q^{\prime}\left(T_{i}, s\right) \frac{\partial^{2}}{\partial a_{i} \partial a_{j}} \varphi\left(x\left(T_{1}\right), x\left(T_{2}\right), \ldots, x\left(T_{k}\right)\right) Q\left(T_{j}, s\right)+ \\
& \quad+\int_{\max (\tau, s)}^{t_{1}}\left[Q^{\prime}(t, \tau) H_{x x}(t) Q(t, s)+Q^{\prime}(h(t), \tau) H_{y x}(t) Q(t, s)+\right. \\
& \left.\quad+Q^{\prime}(t, s) H_{x y}(t) Q(h(t), s)+Q^{\prime}(h(t), \tau) H_{y y}(t) Q(h(t), s)\right] d t .
\end{aligned}
$$

С учетом тождеств (27)-(30) и обозначения (31) неравенство (20) записывается в виде

$$
\begin{aligned}
\int_{t_{0}}^{t_{1}} \int_{t_{0}}^{t_{1}} \delta u^{\prime}(\tau) M(\tau, s) \delta u(s) d s d \tau+ & \\
& +2 \int_{t_{0}}^{t_{1}}\left[\int_{t_{0}}^{t} \delta u^{\prime}(t) H_{u x}(t) Q(t, \tau) \delta u(\tau) d \tau\right] d t+ \\
& +\int_{t_{0}}^{t_{1}}\left[\int_{t_{0}}^{t} \delta u^{\prime}(t) H_{u y}(t) Q(h(t), s) \delta u(s) d s\right] d t+ \\
& +\int_{t_{0}}^{t} \delta u^{\prime}(t) H_{u u}(t) \delta u(t) d t \leqslant 0 .
\end{aligned}
$$

Сформулируем полученный результат.

Теорема. Для оптимальности классической экстремали $u(t)$ в задаче (1)-(4) необходимо, чтобы неравенство (32) выполнялось для всех $\delta и(t) \in \mathbb{R}^{r}$, $t \in T$.

Неравенство (32) является квадратичным интегральным необходимым условием оптимальности второго порядка. Из него можно получить ряд относительно легко проверяемых необходимых условий оптимальности и в частности - аналог условия Лежандра-Клебша и многоточечные необходимые 
условия оптимальности особых в классическом смысле $[17,19,21]$ управлений.

ЗАмЕчАниЕ. Квазиособые управления в задачах оптимального управления системами интегро-дифференциальных уравнений типа Вольтерра исследованы в работе [13].

Заключение. При помощи модификации метода приращений вычислены первая и вторая вариации функционала качества и с их помощью установлены неявные необходимые условия оптимальности первого и второго порядков, позволяющие получить необходимые условия оптимальности, выраженные непосредственно через параметры рассматриваемой задачи.

Конкурирующие интересы. Мы не имеем конкурирующих интересов.

Авторский вклад и ответственность. Все авторы принимали участие в разработке концепции статьи и в написании рукописи. Авторы несут полную ответственность за предоставление окончательной рукописи в печать. Окончательная версия рукописи была одобрена всеми авторами.

Финансирование. Исследование выполнялось без финансирования.

\section{Библиографический список}

1. Алексеев В. М., Тихомиров В. М., Фомин С. В. Оптимальное управление. М.: Наука, 1979. $432 \mathrm{c}$.

2. Appell J. M., Kalitvin A. S., Zabrejko P. P. Partial integral operators and integro-differential equations / Pure and Applied Mathematics. New York: CRC Press, 2000. x+578 pp. doi : 10 . $1201 / 9781482270402$.

3. Volterra V. Theory of functionals and of integral and integro-differential equations. London: Blackie \& Son, 1930. xiv +226 pp.

4. Васильева А. Б., Тихонов А. Н. Интегральные уравнения. М.: Физматлит, 2002. 160 с.

5. Warga J. Optimal control of differential and functional equations. New York, London: Academic Press, 1972. xiii+531 pp. doi: 10.1016/c2013-0-11669-8.

6. Васильев Ф. П. Условия оптимальности для некоторых классов систем, не разрешенных относительно производной // Докл. АН СССР, 1969. Т. 184, № 6. С. 1267-1270.

7. Васильев Ф. П. Об условиях существования седловой точки в детерминированных играх для интегро-дифференциальных систем с запаздыванием нейтрального типа // $A в-$ томат. и телемех., 1972. № 2. С. 40-50.

8. Васильев Ф. П. Об условиях существования седловой точки в детерминированных интегро-дифференциальных играх с запаздыванием при наличии параметров // $K$. вычисл. матем. и матем. физ., 1970. Т. 10, № 1. С. 15-25.

9. Ведь Ю. А., Пахыров 3. Об ограниченности и устойчивости решений интегродифференциальных уравнений с запаздывающим аргументом // Дифферени. уравнения, 1969. Т. 5, № 11. С. 2050-2061.

10. Габасов Р., Кириллова Ф. М. Принцип максимума в теории оптимального управления. Минск: Наука и техника, 1974. 272 с.

11. Габасов Р., Кириллова Ф. М. Качественная теория оптимальных прочессов. М.: Наука, 1971. 508 с.

12. Марданов М. Дж., Гасанов К. К. Условия оптимальности в системах интегродифференциальных уравнений с запаздыванием// Изв. АН Азерб. ССР. Сер. физ.техн. и мат. наук, 1972. № 3. С. 114-119.

13. Mardanov M. J., Mansimov K. B. Necessary optimality conditions of quasi-singular controls in optimal control// Proc. Inst. Math. Mech. of Azerbaijan. Ser. Phys.-Tech. Math. Sci., 2015. vol. 41, no.1. pp. 113-122, Retrieved from http://proc.imm.az/volumes/41-1/ 41-01-12.pdf (December 27, 2017). 
14. Меликов Т. К. Исследование особых прочессов в некоторых оптимальных системах: Автореф. дисс. ... канд. физ.-мат. наук. Баку: Ин-т матем. и механ., 1976. 17 с.

15. Марданов М. Дж. Некоторые вопросы теории оптимального управления в системах интегро-дифференциальных уравнений: Автореф. дисс. ... канд. физ.-мат. наук. Баку: Ин-т матем. и механ., 1976. 21 с.

16. Меликов Т. К., Аббасова С. С. Аналог условия Лежандра-Клебиа в оптимальных системах интегро-дифференииальных уравнений нейтрального типа: Деп. в Азербайджанском научно-исследовательском институте научно-технической информации, № 2222-Аз 94, 1994.

17. Габасов Р., Кириллова Ф. М. Особые оптимальные управления. М.: Наука, 1973. 256 с.

18. Демьянов В. Ф. Условия экстремума и вариационное исчисление. М.: Высш. шк., 2005. $335 \mathrm{c}$.

19. Мансимов К. Б. Особые управления в системах с запаздыванием. Баку: Елм, 1999. $174 \mathrm{c}$.

20. Мансимов К. Б., Марданов М. Дж. Качественная теория оптимального управления системами Гурса-Дарбу. Баку: Элм, 2010. 360 с.

21. Мансимов К. Б. Многоточечные необходимые условия оптимальности особых в классическом смысле управлений в системах с запаздыванием// Дифферени. уравнения, 1985. T. 21, № 3. С. 527-530. 


\title{
MSC: 49K15, 49K21, 45J05, 45D99, 37N40
}

\section{Integral necessary condition of optimality of the second order for control problems described by system of integro-differential equations with delay}

\author{
M. J. Mardanov ${ }^{1}$, K. B. Mansimov ${ }^{2,3}$, N. H. Abdullayeva ${ }^{3}$ \\ 1 Institute of Mathematics and Mechanics, Azerbaijan National Academy of Sciences, \\ 9, Bakhtiyar Vahabzade st., Baku, AZ1141, Azerbaijan. \\ 2 Baku State University, \\ 23, Acad. Zahid Khalilov st., Baku, AZ1148, Azerbaijan. \\ 3 Institute of Control Systems, Azerbaijan National Academy of Sciences, \\ 9, Bakhtiyar Vahabzade st., Baku, AZ1141, Azerbaijan.
}

\begin{abstract}
We consider the optimal control problem that is described by the system of integro-differential equations of the Volterra type with delay and multipoint performance criterion. The first and the second variations of the performance criterion are calculated under the hypothesis that the control domain is open. The necessary condition of the first order optimality in the form analogous to the Euler equations is deduced from the equality of the first variation of performance criterion and zero along the optimal process. Next, the implicit necessary condition of the second order optimality is obtained, which helps to establish rather general but constructively verified necessary condition for the second order optimality. The obtained results are applicable for constructing easy-verifying necessary conditions of optimality for the singular (in the usual sense) controls.
\end{abstract}

Keywords: integro-differential equation of Volterra type, optimal equation, necessary optimality condition in integral form, analog of Euler's equation, classical extreme, necessary second-order optimality condition.

Received: $27^{\text {th }}$ December, $2017 /$ Revised: $14^{\text {th }}$ April, $2018 /$

Accepted: $11^{\text {th }}$ June, 2018 / First online: $1^{\text {st }}$ July, 2018

\section{Research Article}

( )(i) The content is published under the terms of the Creative Commons Attribution 4.0 International License (http://creativecommons.org/licenses/by/4.0/)

Please cite this article in press as:

Mardanov M. J., Mansimov K. B., Abdullayeva N. H. Integral necessary condition of optimality of the second order for control problems described by system of integro-differential equations with delay, Vestn. Samar. Gos. Tekhn. Univ., Ser. Fiz.-Mat. Nauki [J. Samara State Tech. Univ., Ser. Phys. Math. Sci.], 2018, vol. 22, no. 2, pp. 254-268. doi: 10.14498/vsgtu1597 (In Russian).

\section{Authors' Details:}

Misir J. Mardanov (D) http://orcid.org/0000-0003-3901-0719

Dr. Phys. \&. Math. Sci., Professor, Corresponding Members of the Azerbaijan National Academy of Sciences; Director of the Institute ${ }^{1}$; e-mail:misir.mardanov@imm.az

Kamil B. Mansimov (D) http://orcid.org/0000-0002-1518-2279

Dr. Phys. \&. Math. Sci., Professor; Head of Dept.; Dept. of Mathematical Cybernetics ${ }^{2}$; Head of Lab.; Lab. of Control in Complex Dynamic Systems ${ }^{3}$; e-mail: kamilbmansimov@gmail.com

Nisa H. Abdullayeva (1) http://orcid.org/0000-0002-8834-5278

Postgraduate Student; Lab. of Control in Complex Dynamic Systems ${ }^{3}$;

e-mail: kmansimov@mail.ru 
Competing interests. We have no competing interests.

Authors' contributions and responsibilities. Each author has participated in the article concept development and in the manuscript writing. The authors are absolutely responsible for submitting the final manuscript in print. Each author has approved the final version of manuscript.

Funding. The research has not had any funding.

\section{References}

1. Alekseev V. M., Tikhomirov V. M., Fomin S. V. Optimal Control, Contemporary Soviet Mathematics. Boston, Springer, 1987, xiii+309 pp. doi : 10.1007/978-1-4615-7551-1.

2. Appell J. M., Kalitvin A. S., Zabrejko P. P. Partial integral operators and integro-differential equations, Pure and Applied Mathematics. New York, CRC Press, 2000, x+578 pp. doi : 10 . 1201/9781482270402.

3. Volterra V. Theory of functionals and of integral and integro-differential equations. London, Blackie \& Son, 1930, xiv +226 pp.

4. Vasil'eva A. B., Tikhonov A. N. Integral'nye uravneniia [Integral Equations]. Moscow, Fizmatlit, 2002, 160 pp. (In Russian)

5. Warga J. Optimal control of differential and functional equations. New York, London, Academic Press, 1972, xiii+531 pp. doi: 10.1016/c2013-0-11669-8.

6. Vasil'ev F. P. Optimality conditions for some classes of systems not solved with respect to the derivative, Sov. Math., Dokl., 1969, vol.10, pp. 224-227.

7. Vasil'ev F. P. Concerning conditions of existence of a saddle point in determinate games for integro-differential systems with a neutral type delay, Avtomat. $i$ Telemekh., 1972, no. 2, pp. 40-50 (In Russian).

8. Vasil'ev F. P. Conditions for a saddle-point to exist in determinate integro-differential games involving delay and parameters, U.S.S.R. Comput. Math. Math. Phys., 1970, vol.10, no. 1, pp. 17-30. doi: 10.1016/0041-5553(70)90067-4.

9. Ved' Yu. A., Pahyrov Z. The boundedness and stability of the solutions of integro-differential equations with retarded argument, Differ. Uravn., 1969, vol. 5, no. 11, pp. 2050-2061 (In Russian).

10. Gabasov R., Kirillova F. M. Printsip maksimuma v teorii optimal'nogo upravleniia [Maximum Principle in Optimal Control Theory]. Minsk, Nauka i tekhnika, 1974, 272 pp. (In Russian)

11. Gabasov R., Kirillova F. The Qualitative Theory of Optimal Processes, Control and Systems Theory, vol. 3. New York, Basel, Marcel Dekker, 1976, xlvi+640 pp.

12. Mardanov M. J., Gasanov K. K. Optimality conditions for systems of delay integrodifferential equations, Izv. Akad. Nauk Az. SSR, Ser. Fiz.-Tekh. Mat. Nauk, 1972, no.3, pp. 114-119 (In Russian).

13. Mardanov M. J., Mansimov K. B. Necessary optimality conditions of quasi-singular controls in optimal control, Proc. Inst. Math. Mech. of Azerbaijan. Ser. Phys.-Tech. Math. Sci., 2015, vol. 41, no. 1, pp. 113-122, Retrieved from http://proc.imm.az/volumes/41-1/41-01-12. pdf (December 27, 2017).

14. Melikov T. K. Investigation of special processes of some optimal systems, Thesis of Dissertation (Cand. Phys. \& Math. Sci.). Baku, Inst. Math. Mech., 1976, 17 pp. (In Russian)

15. Mardanov M. J. Some issues of theory of optimal controls in the systems of integrodifferential equations, Thesis of Dissertation (Cand. Phys. \& Math. Sci.). Baku, Inst. Math. Mech., 1976, 21 pp. (In Russian)

16. Melikov T. K., Abbasova S. S. An analogue of the Legendre-Clebsch condition in optimal systems of integro-differential equations of neutral type, Preprint, Azerbaijan Research Institute of Scientific and Technical Information, no. 2222-Az 94, 1994 (In Russian).

17. Gabasov R., Kirillova F. M. Osobye optimal'nye upravleniia [Singular Optimal Controls]. Moscow, Nauka, 1973, 256 pp. (In Russian) 
18. Dem'ianov V. F. Usloviia ekstremuma i variatsionnoe ischislenie [Extremum Conditions and Calculus of Variations]. Moscow, Vyssh. shk., 2005, 335 pp. (In Russian)

19. Mansimov K. B. Osobye upravleniia $v$ sistemakh $s$ zapazdyvaniem [Singular Controls in Delay Systems]. Baku, Elm, 1999, 174 pp. (In Russian)

20. Mansimov K. B., Mardanov M. D. Kachestvennaia teoriia optimal'nogo upravleniia sistemami Gursa-Darbu [Qualitative Theory of Optimal Control for Goursat-Darboux Systems]. Baku, Elm, 2010, 360 pp. (In Russian)

21. Mansimov K. B. Multipoint necessary conditions for optimality of controls that are singular in the classical sense in systems with delay, Differ. Uravn., 1985, vol. 21, no. 3, pp. 527-530 (In Russian). 